\title{
Colorectal Cancer: Basic and Translational Research
}

\section{J.J. Zhou S. Zheng}

Department of Surgical Oncology, Second Affiliated Hospital, and The Key Laboratory of Cancer Prevention and Intervention, China National Ministry of Education, Zhejiang University School of Medicine, Hangzhou, China

\section{Key Message}

Translational research has led to significant benefits in the screening and management of patients with colorectal cancer, such as the identification of responders to cetuximab therapy, and the application of microsatellite instability and intrinsic subtyping of colorectal cancers towards personalized therapy.

\section{Practical Implications}

The process of translational research involves four basic strategies: (a) biomarker discovery, (b) identification of the biomarkers in xenografts, (c) population-based verification and (d) clinical validation. Several prognostic biomarkers, namely SPARCL1, HMGA2, RRM2 and SOX2, have been successfully identified following these strategies. To address the problem of treatment failure, therapy should be adapted to the molecular phenotype and colorectal cancer intrinsic subtype of the tumor. A case in point is the presence of mutated KRAS, which can be used to identify non-responders to cetuximab therapy. Likewise, microsatellite instability is a strong predictor of response to fluorouracil-based adjuvant chemotherapy in stage II and III colon cancer. A comprehensive classification of the intrinsic subtypes of colorectal cancer not only enhances our understanding of response to therapy and prognosis, but also enables treatment to be tailored to the individual patient. 


\title{
Key Words
}

Colorectal neoplasms · Therapeutics · Translational medical research

\begin{abstract}
Background: Colorectal cancer (CRC) still accounts for high mortality and morbidity of cancer patients worldwide. Early detection screening and therapeutic management of CRC are still traditional, with some important progress including cetuximab, microsatellite instability and the concept of CRC intrinsic subtypes. Summary: Most achievements are derived from translational cancer research. Translational cancer research attempts to better understand the heterogeneity of human cancer and further turn discoveries into benefits for cancer patients. The four basic strategies of translational research can be depicted as (a) biomarker discovery, (b) identification in xenografts, (c) population-based verification and (d) clinical validation.
\end{abstract}

(C) 2013 S. Karger AG, Basel

\section{Introduction}

Colorectal cancer (CRC) is the third most commonly diagnosed cancer in males and the second in females [1]. The incidence rates of CRC, including Eastern Asia and Eastern Europe, are still increasing, although the United States is the only country with significantly decreasing incidence rates, owing to the sensible practice of CRC detection screening [2]. In addition, current therapeutic managements for CRC are mainly based on surgery, chemotherapy, radiotherapy and molecularly targeted therapy. Molecularly targeted therapy, mostly derived from translational cancer research, has achieved good therapeutic effect. However, the heterogeneity of CRC leads to the same treatment achieving different individual responses. This stresses the need for translational research, especially with respect to discovering the suitable molecular targets for matching different individuals.

The enterprise of translational cancer research was initiated 40 years ago and has always been emphasizing on the bench-to-bedside and bedside-to-bench researches. The ultimate goal of translational cancer research is to better understand the heterogeneity of human cancer and further turn discoveries into benefits for cancer patients [3]. Despite great challenges, progress in the translational research of cancer is constantly emerging. Evidently, from cytotoxic T lymphocyte research to ipilimumab used in metastatic melanoma $[4,5]$ and breast cancer subtype assessed by estrogen receptor, progesterone receptor and HER-2, which proved to be intensely associated with the prognosis and treatment response of breast cancer [6], many novel drugs derived from translational cancer research have come to be the mainstay of clinical cancer treatment. Simultaneously, CRC also witnesses the substantial progress that translational cancer research brings into clinical development.

\section{Basic Strategies in Translational Research}

Translational research is defined as 'the application of a discovery to the practice of medicine' [7]. However, more precisely, the process of translational research can be viewed as a cycle, consisting of several identifiable phases: observation with a bright idea, synthesis with medical knowledge, hypothesis testing and further discovery, and application to clinical testing $[3,7]$. The process of translational research underlines the requirement of clinical application-directed biological research. For another aspect, specifically in CRC translational research, we can depict the basic strategies of translational research into four steps (fig. 1): 
Fig. 1. Four basic strategies of translational research.

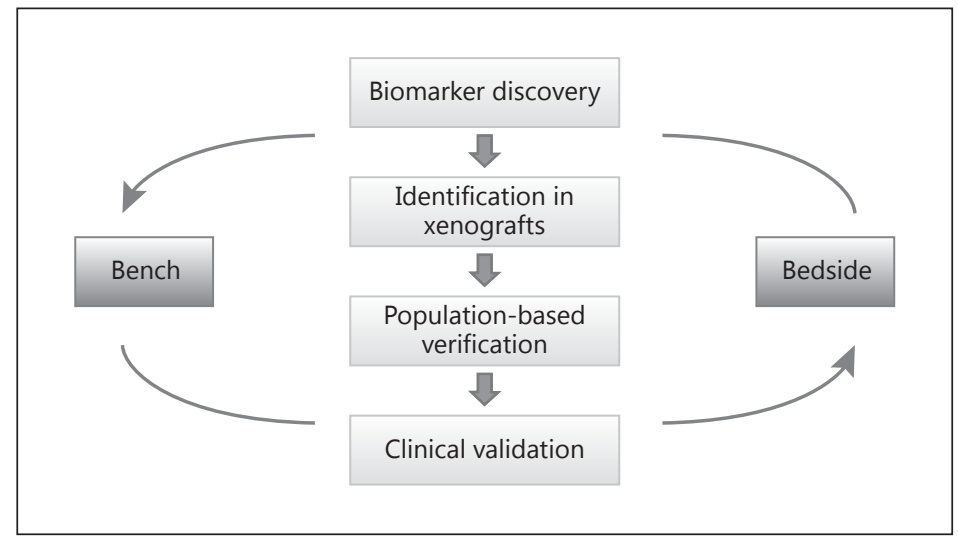

\section{Biomarker Discovery}

Biomarker selection evolves from the comprehensive analysis of cancer biology. To date, some biomarkers are commonly selected from genome screening. A comprehensive genomescale analysis of 276 samples was conducted to characterize somatic alterations in colorectal carcinoma by the Cancer Genome Atlas Network [8], suggesting a great number of potential biomarkers involved in the molecular biological process in colorectal carcinogenesis, such as high microsatellite instability (MSI), MLH1 silencing, somatic mismatch repair gene and polymerase e mutations, APC, TP53, SMAD4, PIK3CA and KRAS mutation.

\section{Identification in Xenografts}

After the discovery of the biomarker of interest, we have to identify its biological behavior and function in xenografts both in vitro and in vivo. The cell line-propagated xenografts cannot completely resemble the corresponding human tumor for one important reason: they lack the cell stroma to resemble the microenvironment of the true tumor cells. Much research has proved the substantial role the stroma plays in carcinogenesis [9], which strongly suggests the importance of xenograft identification in vivo. Most of the mouse models for CRC in the past were chemically induced, whereas now transgenic mouse models are used more frequently in CRC research.

\section{Population-Based Verification}

For CRC, human specimens from biopsy or surgical resection are suitable samples for population-based verification. High-quality biospecimens in tissue banks, combined with the corresponding bioinformatics and elaborate medical records, are crucial resources for the primary verification of the identification results from the lab [10].

\section{Clinical Validation}

The final goal of translational cancer research is to translate research discoveries into clinical practice, which ultimately benefits the patients. Therefore, clinical validation is the gold standard to determinate the triumph of the translational research. For clinical cancer research, survival has always been the primary endpoint for consideration. Besides, we have to pay more attention to the life quality of cancer patients, and the European Organisation for Research and Treatment of Cancer (EORTC) provides quality of life questionnaires for assessment [11]. 


\section{Discovery of Molecular Biomarkers and Validation with Multiple Clinical Centers}

Certain experience has been accumulated which followed the basic strategies, starting from the discovery of biomarkers to the identification of biological function and validation with multiple clinical centers afterwards.

$\mathrm{Hu}$ et al. [12] selected the secreted protein, acidic and rich in cysteine-like 1 (SPARCL1) from the genomic screening of two groups of primary CRC with or without liver metastasis. In both in vitro and vivo studies, the ectopic expression of SPARCL1 was identified as a tumor suppressor. A retrospective outcome study [the $\mathrm{COH}$ set (222 CRCs) and the ZJU set (412 CRCs)] then also revealed that the role of SPARCL1 was associated with better overall survival, indicating the potential of SPARCL1 as a prognosis marker.

Wang et al. [13] focused on the high-mobility group A2 (HMGA2), an oncofetal protein, of which it was proved that its overexpression is associated with CRC metastasis and reduced survival rates. This retrospective multi-center study was conducted with 89 surgical CRC specimens of the training set and 191 of the validation set. Therefore, HMGA2 may potentially serve as a biomarker for aggressive CRC with poor survival.

Liu et al. [14] investigated the ribonucleotide reductase small subunit M2 (RRM2), whose overexpression was proved to dramatically enhance the ability of proliferation and invasion of cancer cells. The retrospective multi-center ( $\mathrm{COH}$ set and ZJU set) outcome study was conducted to investigate the relevance of RRM2 and CRCs, which account for 437 cases in total. In this study, RRM2 was proved to be an independent prognostic factor predicting poor survival of CRC.

Han et al. [15] conducted research on SOX2, a member of the SOX family gene that plays a critical role in embryonic stem cells. Silencing of SOX2 was biologically investigated to induce mesenchymal epithelial transition. Furthermore, SOX2 activity was closely associated with the WNT pathway, and immunofluorescence cell staining analysis of 44 CRC specimens suggested the potential possibility of SOX2 as a prognosis marker for CRC metastasis.

These four studies were in accordance with the four basic strategies of translational research, including population-based verification and validation by two clinical centers. Namely, before clinically consented and accepted, further validation by multiple clinical centers is still needed.

\section{Individualized Therapy and Tumor Heterogeneity}

Although there are appropriate therapies for CRC, treatment failure still remains as the primary intractable problem. The main reasons for treatment failure are heterogeneity of the tumor. Therefore, the conception of individualized therapy based on specific molecular phenotype and furthermore CRC intrinsic subtype will probably solve the problem.

\section{Specific Molecular Phenotypes}

KRAS. Cetuximab, a monoclonal antibody anti-epidermal growth factor receptor (antiEGFR), was one of the efficient drugs for metastatic CRC. However, the mechanism of the different clinical responses to cetuximab remained unknown until the KRAS mutation was found [16]. KRAS, a member of the RAS proteins family, which belongs to the guanosine-5-triphosphatase (GTase) superfamily, is the important downstream of EGFR in the EGFR signaling cascade. Both retrospective and prospective clinical studies [17-21] proved the predictive value of KRAS mutation, revealing that only patients with a tumor bearing wild-type KRAS benefit from cetuximab, which is not the case for mutated KRAS [22, 23].

MSI. MSI, the phenotype resulting from DNA mismatch repair, is defined as the instability of simple repeated DNA sequences named microsatellites. It was commonly believed to be a 
second mutational pathway for colorectal carcinogenesis, accounting for approximately $15 \%$ of sporadic CRCs [24, 25]. However, the prominent translational significance of MSI is that it has been clinically proved to be a predictor of the benefit of fluorouracil-based adjuvant chemotherapy in stage II and III colon cancer [26, 27]. Mismatch repair status assessment has been required to be taken into consideration before fluorouracil-based chemotherapy treatment decision making, which is already in the NCCN guidelines (version 3.2013).

\section{Colorectal Cancer Intrinsic Subtypes and Individualized Therapy}

As specific molecular phenotypes discovered in CRC such as KRAS and MSI accumulate, a comprehensive classification for the intrinsic subtypes of CRC is essentially needed to gain better understanding of the biological network of CRC. The significant associations between intrinsic subtype and clinical outcomes arouse many concerns on the research for CRC intrinsic subtypes or 'molecular classification'.

Sadanandam et al. [28] first clustered two published gene expression data sets (GSE13294 and GSE14333) of resected primary CRCs, defining five subtypes by specific gene expressions: goblet-like, enterocyte, stem-like, inflammatory and transit-amplifying. Due to the differences in cetuximab sensitivity and the analysis of the related gene expression level such as the EGFR ligands epiregulin and amphiregulin, and the MET regulator filamin A, the transitamplifying subtype was designated into cetuximab-sensitive and -resistant, actually increasing the number of subtypes to six. Similarly, the study also confirmed the significant association between response signature of the chemotherapy regimen FOLFIRI and different subtypes, in which stem-like subtype was the preferentially suggested FOLFIRI chemotherapy in both adjuvant and metastatic settings. Anatomically, the five subtypes are located in different regions in colon crypts from base to top, while phenotypically they are distinct in disease-free survival after surgical resection, which has an important prognostic implication.

Another gene expression profile involving over 1,100 individuals was carried out by De Sousa E Melo et al. [29], recognizing three molecularly distinct subtypes. Colon cancer subtype 1 (CCS1) and CCS2 were defined in accordance with previous identification, respectively associated with CIN and MSI (chromosomal-instable and microsatellite-instable cancers). The CCS3 tumor was largely microsatellite-stable and contains more $\mathrm{CpG}$ island methylator phenotypepositive carcinomas, with a significantly poor prognosis. Otherwise, the CCS3 tumor was highly related to serrated adenomas and proved to be resistant to cetuximab therapy.

Recently, an ongoing study for molecular classification of CRC into subtypes revealed that CRC consists of at least three major intrinsic subtypes ( $\mathrm{A}, \mathrm{B}$ and $\mathrm{C}$ type). The classification is mainly based on three biological hallmarks of the tumor, including epithelial-to-mesenchymal transition, deficiency in mismatch repair genes that result in a high mutation frequency associated with MSI, and cellular proliferation [30].

All the studies uncover the potential possibility that the intrinsic subtypes of CRC are significantly associated with metastasis, prognosis and chemotherapy response, directing different treatment strategies. It meets the demand of individualized therapy for CRC, due to the clinical and biological heterogeneity of CRC. In essence, the clinical diversities derive from the intrinsic diverse molecular classification, which underlines the important role of the intrinsic classification in directing clinical treatments.

\section{Perspectives and Conclusion}

It is unambiguous that translational research is the right direction and the mainstay for the clinical and biological development of CRC treatment. However, our progress achieved from translational research is still slow and limited. It enhances the requirement of encour- 
aging an intense academic connection between the clinical surgeon, the physician and biological researchers, effectively operating the cycle of bench-to-bedside and bedside-tobench. Individualized therapy intensely relies on translational research, which probably eliminates the heterogeneity-derived treatment failure of CRC. Otherwise, as carcinogenesis is a redundant complex network involving an enormous number of genes, RNAs, microRNAs, proteins and metabolic molecules etc., we have to conduct the molecular classification of $\mathrm{CRC} /$ other cancers referring to different molecular events, and this could further guide the clinic practice of comprehensive perspective.

\section{References}

1 Jemal A, et al: Global cancer statistics. CA Cancer J Clin 2011;61:69-90.

-2 Edwards BK, et al: Annual report to the nation on the status of cancer, 1975-2006, featuring colorectal cancer trends and impact of interventions (risk factors, screening, and treatment) to reduce future rates. Cancer 2010;116:544-573.

3 Hait WN: Forty years of translational cancer research. Cancer Discov 2011;1:383-390.

4 Hodi FS, et al: Improved survival with ipilimumab in patients with metastatic melanoma. N Engl J Med 2010; 363:711-723.

5 Robert C, Thomas L, Bondarenko I, O’Day S, Weber J, Garbe C, Lebbe C, Baurain JF, Testori A, Grob JJ, Davidson N, Richards J, Maio M, Hauschild A, Miller WH, Gascon P, Lotem M, Harmankaya K, Ibrahim R, Francis S, Chen TT, Humphrey R, Hoos A, Wolchok JD: Ipilimumab plus dacarbazine for previously untreated metastatic melanoma. N Engl J Med 2011;364:2517-2526.

6 Nguyen PL, et al: Breast cancer subtype approximated by estrogen receptor, progesterone receptor, and HER-2 is associated with local and distant recurrence after breast-conserving therapy. J Clin Oncol 2008;26: 2373-2378.

7 Hait WN: Translating research into clinical practice: deliberations from the American Association for Cancer Research. Clin Cancer Res 2005;11:4275-4277.

-8 Cancer Genome Atlas Network: Comprehensive molecular characterization of human colon and rectal cancer. Nature 2012;487:330-337.

-9 Hanahan D, Weinberg RA: Hallmarks of cancer: the next generation. Cell 2011;144:646-674.

10 Suh KS, et al: Tissue banking, bioinformatics, and electronic medical records: the front-end requirements for personalized medicine. J Oncol 2013;2013:368751.

11 Fayers PM, et al: The EORTC QLQ-C30 Scoring Manual, ed 3. Available at: http://groups.eortc.be/qol/eortcqlq-c30 (accessed May 10, 2013).

$\checkmark 12 \mathrm{Hu} \mathrm{H}$, et al: Secreted protein acidic and rich in cysteines-like 1 suppresses aggressiveness and predicts better survival in colorectal cancers. Clin Cancer Res 2012;18:5438-5448.

13 Wang X, et al: Overexpression of HMGA2 promotes metastasis and impacts survival of colorectal cancers. Clin Cancer Res 2011;17:2570-2580.

14 Liu X, et al: Ribonucleotide reductase small subunit M2 serves as a prognostic biomarker and predicts poor survival of colorectal cancers. Clin Sci 2013;124:567-578.

15 Han X, et al: Silencing SOX2 induced mesenchymal-epithelial transition and its expression predicts liver and lymph node metastasis of CRC patients. PLoS One 2012;7:e41335.

16 Lièvre A, Bachet JB, Le Corre D, et al: KRAS mutation status is predictive of response to cetuximab therapy in colorectal cancer. Cancer Res 2006;66:3992-3995.

17 Di Fiore F, et al: Clinical relevance of KRAS mutation detection in metastatic colorectal cancer treated by Cetuximab plus chemotherapy. Br J Cancer 2007;96:1166-1169.

18 Khambata-Ford S, et al: Expression of epiregulin and amphiregulin and K-ras mutation status predict disease control in metastatic colorectal cancer patients treated with cetuximab. J Clin Oncol 2007;25:3230-3237.

19 Bokemeyer C, et al: Fluorouracil, leucovorin, and oxaliplatin with and without cetuximab in the first-line treatment of metastatic colorectal cancer. J Clin Oncol 2008;27:663-671.

20 Hamilton SR: Targeted therapy of cancer: new roles for pathologists in colorectal cancer. Mod Pathol 2008; 21:S23-S30.

-21 Lièvre A, et al: KRAS mutations as an independent prognostic factor in patients with advanced colorectal cancer treated with cetuximab. J Clin Oncol 2008;26:374-379.

-22 Karapetis CS, Khambata-Ford S, Jonker DJ, O'Callaghan CJ, Tu D, Tebbutt NC, Simes RJ, Chalchal H, Shapiro JD, Robitaille S, Price TJ, Shepherd L, Au HJ, Langer C, Moore MJ, Zalcberg JR: K-ras mutations and benefit from cetuximab in advanced colorectal cancer. N Engl J Med 2008;359:1757-1765.

-23 Jimeno A, Messersmith WA, Hirsch FR, Franklin WA, Eckhardt SG: KRAS mutations and sensitivity to epidermal growth factor receptor inhibitors in colorectal cancer: practical application of patient selection. J Clin Oncol 2009;27:1130-1136. 
-24 Gryfe R, Kim H, Hsieh ET, Aronson MD, Holowaty EJ, Bull SB, Redston M, Gallinger S: Tumor microsatellite instability and clinical outcome in young patients with colorectal cancer. N Engl J Med 2000;342:69-77.

25 Markowitz SD, Bertagnolli MM: Molecular origins of cancer: molecular basis of colorectal cancer. N Engl J Med 2009;361:2449-2460.

-26 Ribic CM, Sargent DJ, Moore MJ, Thibodeau SN, French AJ, Goldberg RM, Hamilton SR, Laurent-Puig P, Gryfe R, Shepherd LE, Tu D, Redston M, Gallinger S: Tumor microsatellite-instability status as a predictor of benefit from fluorouracil-based adjuvant chemotherapy for colon cancer. N Engl J Med 2003;349:247-257.

27 Sargent DJ, et al: Defective mismatch repair as a predictive marker for lack of efficacy of fluorouracil-based adjuvant therapy in colon cancer. J Clin Oncol 2010;28:3219-3226.

28 Sadanandam A, et al: A colorectal cancer classification system that associates cellular phenotype and responses to therapy. Nat Med 2013;19:619-625.

29 De Sousa E Melo F, et al: Poor-prognosis colon cancer is defined by a molecularly distinct subtype and develops from serrated precursor lesions. Nat Med 2013;19:614-618.

30 Simon I, et al: Association of colorectal cancer intrinsic subtypes with prognosis, chemotherapy response, deficient mismatch repair, and epithelial to mesenchymal transition (EMT). J Clin Oncol 2012;30(suppl 34):abstr 333. 